\title{
Les cellules souches embryonnaires : un modèle cellulaire pour l'étude de la différenciation cardiaque normale et pathologique
}

$\mathbf{L}$ 'existence de lignées cellulaires a été un avantage déterminant pour l'analyse et la compréhension des mécanismes de régulation de l'expression des gènes qui interviennent lors de la différenciation des muscles striés du squelette. L'absence de tels systèmes cellulaires pour le muscle cardiaque explique le décalage qui existe dans nos connaissances entre ces deux systèmes.

Les cellules souches embryonnaires (cellules ES), ainsi que les cellules de carcinome embryonnaire (cellules EC) sont capables, en culture, de se différencier en de multiples types cellulaires et en particulier en myocytes cardiaques [1]. Au cours des dix dernières années, de nombreux groupes ont utilisé ces systèmes pour étudier divers aspects concernant la différenciation cardiaque et ont établi que leur différenciation en myocytes cardiaques reproduit une série de transitions programmées de l'expression des gènes $[2,3]$.

Les cellules souches embryonnaires constituent un système modèle de différenciation cardiaque en culture

Les cellules ES dérivent de la masse cellulaire interne des blastocystes de souris $[1,4,5]$. Elles sont maintenues à l'état indifférencié par culture sur une couche de cellules nourricières (fibroblastes embryonnaires de souris) ou en présence de certains facteurs de croissance. Ces cellules peuvent être induites à se différencier in vitro et de nombreuses études ont montré qu'il se forme alors des dérivés provenant des trois couches cellulaires primitives: l'endoderme, l'ecto- les nombreux phénotypes cellulaires présents, on observe des ensembles de cellules qui présentent des contractions rythmiques spontanées. L'observation de ces cellules au microscope électronique montre la présence de filaments organisés en sarcomères caractéristiques.

Les cellules EC représentent un système très voisin de celui des cellules ES. Ces cellules EC sont les cellules souches de tumeurs malignes particulières que sont les tératocarcinomes. Exactement comme pour les cellules ES, les cellules EC peuvent être induites à se différencier en culture et, dans ces conditions, de nombreux phénotypes cellulaires apparaissent qui appartiennent aux trois feuillets embryonnaires primitifs [7]. Les modalités pratiques d'induction de la différenciation des cellules EC ainsi que les phénotypes différenciés obtenus étant très voisins de ceux décrits pour les cellules ES, dans la suite de cette présentation, nous présenterons indistinctement des résultats obtenus avec des cellules ES ou des cellules EC.

\section{Différenciation des cellules ES en culture}

Le principe de la différenciation des cellules ES et des cellules EC est schématiquement décrit sur la figure 1 . Pour que les cellules ES se différencient, il est nécessaire qu'elles puissent former des structures tridimensionnelles appelées corps embryoïdes. Ceux-ci ne se forment que si les cellules sont placées dans des conditions où elles ne peuvent pas s'attacher au plastique de la boîte de Pétri, soit que la culture ait lieu dans des boîtes de type bactériolo- gique, soit que les cellules soient cultivées dans un système de "gouttes pendantes". La première méthode ne permet pas de contrôler le nombre de cellules qui participent à la formation des agrégats alors que ce nombre est parfaitement contrôlé dans la deuxième méthode, c'est donc cette dernière que l'on utilise de préférence.

Dans une première étape, des gouttes de $20 \mu \mathrm{l}$ de milieu de culture contenant 400 à 600 cellules ES sont déposées sur la face interne du couvercle d'une boîte de Pétri qui est ensuite remis en position normale. Au bout de 48 heures, les cellules se sont rassemblées au fond de la goutte, elles se sont agrégées et ont formé un corps embryoïde. Les corps embryoïdes sont alors collectés et mis en culture dans des boîtes de type bactériologique pendant 4 jours pour leur permettre de continuer leur développement. Enfin, les corps embryoïdes sont prélevés individuellement et ensemencés dans des plaques à puits multiples. Ils vont alors adhérer à la surface, la différenciation cellulaire va se poursuivre et les premières zones battantes sont généralement détectées dès ce même jour. Les corps embryoïdes différenciés peuvent être maintenus en l'état pendant de très nombreux jours mais on peut aussi repérer les zones battantes, les microdisséquer sous une loupe binoculaire, dissocier les cellules à l'aide d'un traitement à la collagénase et les réensemencer afin d'obtenir des cardiomyocytes isolés.

Il va de soi que les cardiomyocytes ne sont pas les seuls types de cellules différenciées obtenus et l'on observe la présence de phénotypes cellulaires dérivant des trois feuillets primitifs. Entre autres, et selon les conditions 


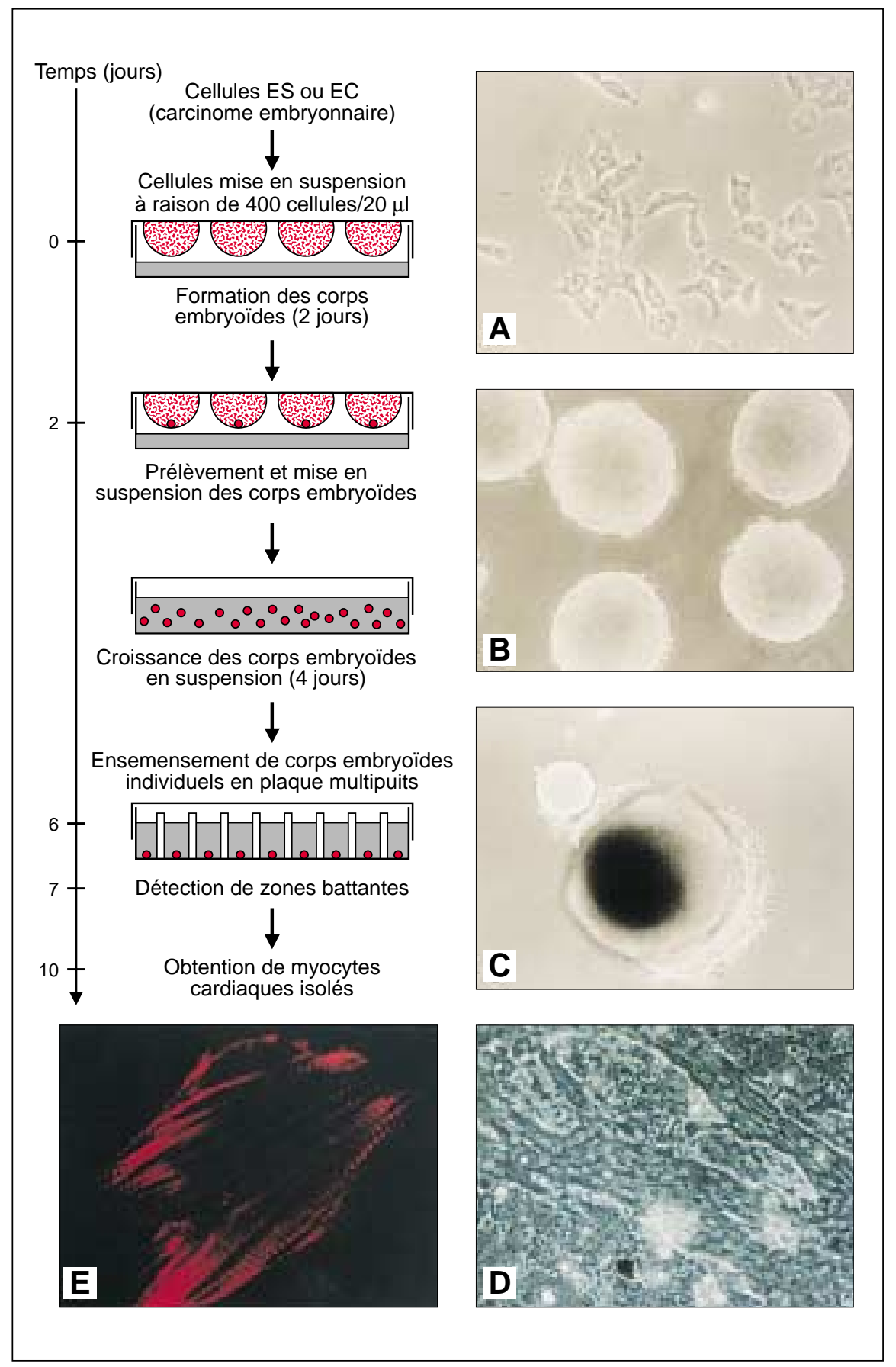

Figure 1. Protocole de différenciation des cellules ES ou des cellules de carcinome embryonnaire. A. Cellules de carcinome embryonnaire indifférenciées. B. Corps embryoïdes en suspension. C. Corps embryoïde attaché. D. Aspect d'une zone battante. E. Cardiomyocytes isolés d'une zone battante, les cellules sont marquées à l'aide d'un anticorps dirigé contre la chaîne lourde de la myosine $\alpha$.

de culture utilisées (nombre de cellules mises dans les gouttes pendantes, acide rétinoïque en concentration variable, etc.), se trouvent des

\section{Expression}

des marqueurs cardiaques

La démonstration de l'utilité potentielle de ce système réside dans la caractérisation des gènes dont l'expression est activée au cours du processus de différenciation. C'est ainsi que de nombreuses protéines cardiaques ont pu être identifiées dans les corps embryoïdes différenciés: les résultats présentés dans le Tableau I proviennent essentiellement des travaux des laboratoires de Ken Chien (La Jolla, CA, USA) [8], de Joseph Metzger (Ann Arbor, MI, USA) [9-11], de Thomas Doetschman (Cincinnati, OH, USA) [12, 13] et de nos propres résultats non publiés. Qui plus est, le profil d'expression de ces protéines récapitule, plus ou moins exactement, les profils d'expression connus pour la différenciation des cellules cardiaques in vivo. C'est ainsi qu'on a pu montrer que l'apparition de la chaîne lourde de la myosine $\beta$ précède celle de la myosine $\alpha[9,14]$. Pour notre part, utilisant la lignée P19 de cellules EC, nous avons observé un décalage d'une journée entre la détection de la myosine $\beta$ par rapport à la myosine $\alpha$ et ce n'est que lorsque les zones battantes apparaissent que les deux chaînes lourdes sont présentes ensemble.

De la même manière, le groupe de Metzger a montré une transition au cours du temps entre les isoformes de troponine I: les zones battantes jeunes expriment préférentiellement l'isoforme de muscle squelettique lent alors que les zones battantes tardives expriment l'isoforme cardiaque [11]. Des variations en fonction du temps de différenciation des corps embryoïdes sont également observées au niveau de l'ultrastructure des cardiomyocytes. C'est ainsi qu'au début de la différenciation, les zones battantes contiennent des myofibrilles organisées en faisceaux irréguliers et en paquets de fibres parallèles et ce n'est que plus tardivement que l'on peut observer des myofibrilles organisées en sarcomères [15] (figure 2). Enfin, des études électrophysiologiques ont clairement indiqué que les cardiomyocytes isolés de zones battantes expriment toute une série 
Tableau I

\section{CONSTITUANTS DONT LA SYNTHĖSE A ÉTÉ DÉTECTÉE DANS LES CARDIOCYTES PROVENANT DE CORPS EMBRYOÏDES DIFFÉRENCIÉS}

\section{Facteurs de transcription}

Nkx 2.5

GATA 4

MEF 2C

MHox

\section{Composants des filaments épais}

Chaîne lourde de la myosine $\beta$

Chaîne lourde de la myosine $\alpha$

Chaîne légère régulatrice de la myosine ventriculaire (MLC2v)

Chaîne légère essentielle de la myosine ventriculaire (MLC1v)

Chaîne légère essentielle de la myosine des oreillettes (MLC1a)

Protéine C cardiaque

\section{Composants des filaments fins}

$\alpha$ actine cardiaque

$\alpha$ tropomyosine

Troponine C cardiaque

Troponine I cardiaque

Troponine I de muscle squelettique lent

Troponine T cardiaque

\section{Autres composants}

$\alpha$ actinine

Connexine 43

Desmine

Facteur natriurétique auriculaire

Phospholamban

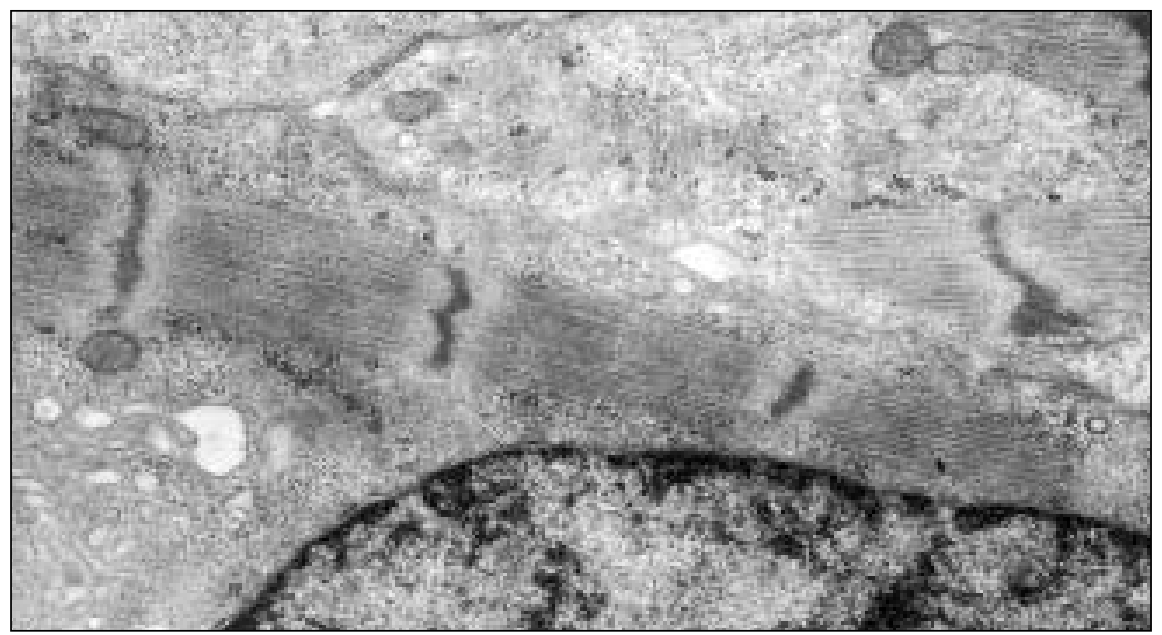

Figure 2. Observation en microscopie électronique de structures sarcomériques dans des cardiomyocytes présents au sein d'une zone battante. de canaux: le profil d'expression de ces canaux évolue avec le temps de différenciation, la panoplie de canaux exprimée par ces cardiomyocytes diffère d'une cellule à l'autre; ainsi, dans une zone battante on peut distinguer des cardiomyocytes ayant des propriétés de cellules nodales, de cellules auriculaires et de cellules ventriculaires [16].

\section{Limitation du système}

S'il n'y a aucun problème pour reconnaître les cardiomyocytes issus des corps embryoïdes, à des stades tardifs de différenciation, du fait de leur contenu typique en matériel myofibrillaire, il n'en va pas de même pour les cardiomyocytes précoces. Il s'en suit que, jusqu'à présent, les études électrophysiologiques ont été exclusivement réalisées sur des cellules présentant des contractions spontanées ce qui a eu pour effet d'éliminer de ces études des cardiomyocytes précoces incapables de se contracter. Pour pallier cet inconvénient, différents systèmes ont été imaginés.

Le premier a consisté à construire des cellules ES exprimant de manière stable un gène de résistance à un antibiotique sous le contrôle d'un promoteur spécifique des cellules cardiaques. Ainsi, l'application de l'antibiotique après le début de la différenciation va provoquer la mort des types cellulaires autres que les cardiomyocytes. L'inconvénient de ce modèle réside dans le temps nécessaire pour que l'antibiotique soit efficace ce qui ne permet pas d'isoler aisément les cardiocytes précoces. Ce système a néanmoins été utilisé pour obtenir des myocytes cardiaques qui ont été ensuite utilisés dans des expériences de greffe cardiaque chez l'animal [17].

Une amélioration a consisté à produire des cellules ES exprimant un colorant vital de manière sélective dans les cardiocytes. Metzger et al. ont utilisé le gène de la $\beta$-galactosidase sous le contrôle du promoteur $\mathrm{du}$ gène humain codant pour l' $\alpha$ actine cardiaque [10]. Le défaut de ce système réside dans la faible sensibilité de la détection de la $\beta$-galactosidase comme colorant vital. Pour 
notre part nous avons tiré avantage de l'extrême sensibilité de la détection de la protéine verte fluorescente (GFP). Nous avons construit des cellules P19 contenant le gène GFP sous le contrôle du promoteur du gène de souris codant pour l' $\alpha$-actine cardiaque. La figure 3 montre un corps embryoïde avec une zone battante: seule cette zone synthétise la GFP. La même figure montre qu'après isolement, les cellules qui produisent la GFP synthétisent bien la myosine cardiaque. L'avantage de ce système tient au fait qu'il est possible d'isoler des cardiomyocytes, à quelque stade que ce soit, à l'aide d'un trieur de cellules. On peut ainsi disposer d'une population pure de cardiocytes, indépendamment de leur capacité de se contracter et à un stade précoce.

La limitation la plus sérieuse concerne l'organisation structurale du sarcomère. En effet, tous les résultats montrent que la formation de la bande $\mathrm{M}$ et des tubules $\mathrm{T}$ est incomplète. La différenciation cardiaque n'atteint donc, probablement, qu'un stade embryonnaire tardif.
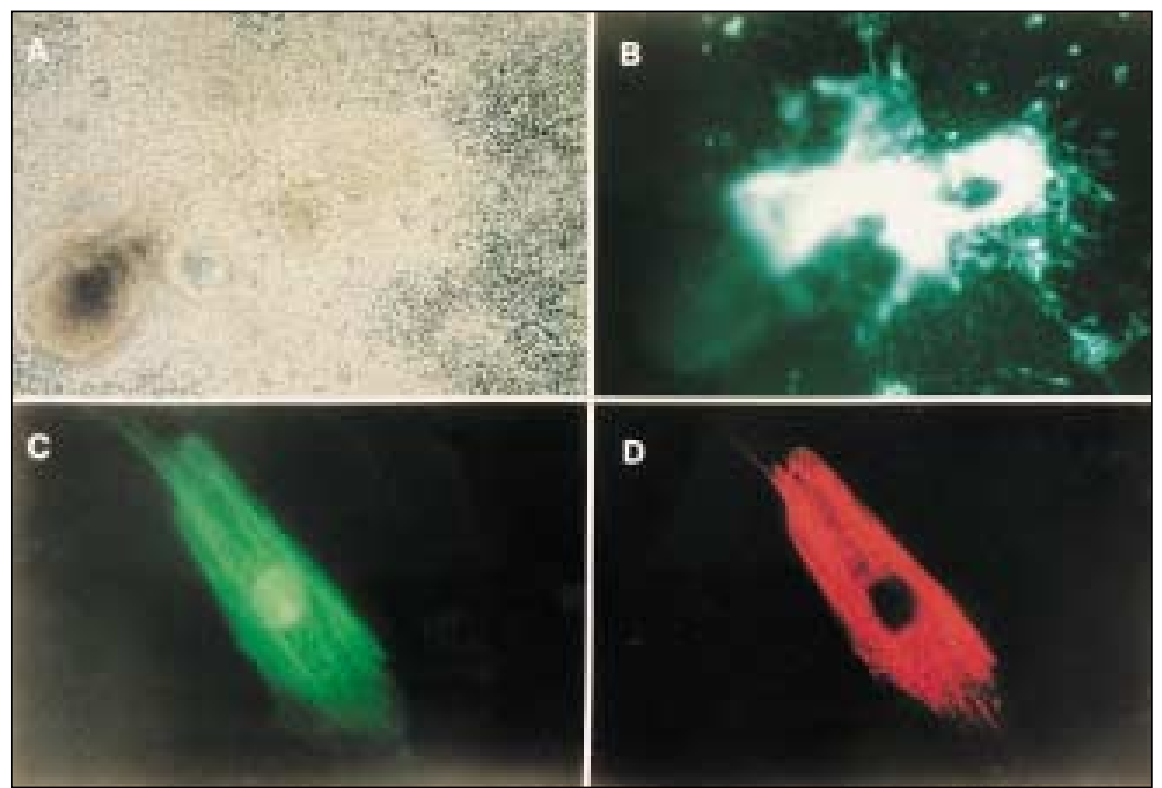

Figure 3. Analyse d'une zone battante provenant d'un corps embryoïde obtenu à partir de cellules de carcinome embryonnaire exprimant le gène GFP sous le contrôle du promoteur du gène de l' $\alpha$-actine cardiaque. A. Corps embryoïde observé au microscope à contraste de phase. B. Le même observé au microscope à fluorescence. C. Cellule isolée de la zone battante et synthétisant la GFP. D. Même cellule après marquage avec un anticorps dirigé contre la chaîne lourde de la myosine $\alpha$. ou l'inactivation d'un gène cardiaque. Ainsi, dans le cas d'une affection comme la cardiomyopathie hypertrophique familiale, on sait que les gènes responsables codent tous pour des protéines de l'appareil contractile (voir dans ce numéro, p. 1054). Il est alors tentant de penser que si l'on peut faire exprimer l'une quelconque de ces mutations par des cardiomyocytes provenant de cellules ES, on devrait pouvoir disposer d'un système permettant d'établir la nature moléculaire du défaut primaire de contraction. C'est ce que notre laboratoire a entrepris de réaliser avec l'un des gènes impliqués, $M y B P-C 3$, qui code pour l'isoforme cardiaque de la protéine $\mathrm{C}$ (voir dans ce numéro, p. 1059) et nos premiers résultats montrent que l'inactivation d'un des allèles de ce gène se traduit par un retard dans l'apparition de l'activité contractile des zones cardiaques au sein des corps embryoïdes. Une mise en garde s'impose toutefois quant à certaines conclusions que l'on pourrait être amené à tirer avec ce système. Ainsi, dans le cas du muscle squelettique, l'inactivation des deux allèles du gène de la desmine entraîne l'absence de cellules musculaires squelettiques dans les corps embryoïdes différenciés en culture [18] alors que cette même inactivation ne se traduit par aucune perturbation de la myogenèse chez les embryons de souris homozygotes [19, 20]. De la même manière, l'inactivation ou l'inhibition de l'expression du gène codant pour GATA-4 se traduit par l'inhibition de la différenciation des cellules P19 en cardiocytes [21] alors que, chez l'animal, l'inactivation du gène n'a aucun effet sur l'apparition des cardiocytes mais se traduit par un défaut de la morphogenèse $d u$ cour [22, 23]. S'il n'y a actuellement aucune explication permettant de rendre compte du désaccord dans le premier exemple, il semble qu'en ce qui concerne l'inactivation de GATA 4, la différence de résultat soit liée au fait que dans les cellules P19 seul GATA 4 est exprimé alors que, dans l'embryon, l'expression de GATA 5 joue vraisemblablement un rôle compensateur, au moins lors des premières étapes de différenciation des cellules cardiaques. 
Bien d'autres applications sont possibles pour le système de cellules ES. L'une d'entre elles, particulièrement intéressante, a récemment fait l'objet d'un article de revue [24]. Il s'agit d'une approche qui utilise les cellules ES pour étudier l'effet d'une mutation létale sur l'induction et l'expression d'un phénotype différencié. C'est ainsi que le groupe d'Anna Wobus (Gatersleben, Allemagne) a montré que des cellules ES dont les deux allèles du gène codant pour l'intégrine $\beta 1$ sont inactivés présentent un défaut dans la cinétique d'apparition du phénotype cardiaque. Un tel résultat ne pouvait être obtenu chez l'animal dans la mesure où l'inactivation des deux allèles de ce gène est létale [25].

Par ailleurs, ces cellules se prêtent parfaitement bien à des études visant à comprendre le processus d'engagement des cellules primitives dans le lignage cardiaque ou celui de la régulation de l'expression des gènes cardiaques. En particulier, il est possible d'envisager d'utiliser les cellules ES comme système préliminaire ou éventuellement alternatif à l'utilisation de souris transgéniques pour identifier et caractériser les éléments régulateurs des promoteurs qui contrôlent l'expression spécifique d'un gène dans les cellules cardiaques.

Il va de soi que le système des cellules ES n'est pas limité à l'étude de la différenciation des cellules cardiaques et il est probable qu'il se révélera un système de choix pour l'étude d'autres types cellulaires comme, par exemple, les cellules nerveuses ou les cellules du muscle lisse

\section{Florence Loubet}

Étudiante en doctorat.

\section{Marc Y. Fiszman}

Directeur de recherche à l'Inserm.

Inserm U. 153, Institut de myologie, Groupe hospitalier Pitié-Salpêtrière, 47, boulevard de l'Hôpital, 75651 Paris Cedex 13, France.

\section{Remerciements}

Les auteurs remercient Mesdames Danièle Thiesson et Josette Destombes (Ura 1448) pour l'analyse des corps embryoïdes en microscopie électronique.

\section{RÉFÉRENCES}

1. Doetschman TC, Eistetter H, Katz M, Schmidt W, Kemler R. The in vitro development of blastocyst-derived embryonic stem cell lines: formation of visceral yolk sac, blood islands and myocardium. I Embryol Exp Morphol 1985 ; 87: 27-45.

2. Hescheler J, Fleischmann BK, Lentini S, Maltsev VA, Rohwedel J, Wobus AM, Addicks K. Embryonic stem cells : a model to study structural and functional properties in cardiomyogenesis. Cardiovasc Res 1997; 36 : $149-62$.

3. Metzger JM, Samuelson LC, Rust EM, Westfall MV. Embryonic stem cell cardiogenesis. Applications for cardiovascular research. Trends Cardiovasc Med 1997; 7: 63-8.

4. Martin GR. Isolation of a pluripotent cell line from early mouse embryos cultured in medium conditioned by teratocarcinoma stem cells. Proc Natl Acad Sci USA 1981; 78 : 7634-8.

5. Evans MJ Kaufman MH. Establishment in culture of pluripotential cells from mouse embryos. Nature 1981; 292: 154-6.

6. Wobus AM, Holzhausen H, Jäkel P, Schöneich J. Characterization of a pluripotent stem cell line derived from a mouse embryo. Exp Cell Res 1984; 152: 212-9.

7. Martin GR. Teratocarcinomas as a model system for the study of embryogenesis and neoplasia. Cell 1975 ; 5 : 229-43.

8. Miller-Hance WC, LaCorbière M, Fuller SJ, Evans SM, Lyons G, Schmidt C, Robbins J, Chien KR. In vitro chamber specification during embryonic stem cell cardiogenesis. Expression of the ventricular myosin light chain-2 gene is independent of heart tube formation. J Biol Chem 1993; 268: 25244-52.

9. Metzger JM, Lin WI, Johnston RA, Westfall MV, Samuelson LC. Myosin heavy chain expression in contracting myocytes isolated during embryonic stem cell cardiogenesis. Circ Res 1995; 76: 710-9.

10. Metzger JM, Lin W, Samuelson LC. Vital staining of cardiac myocytes during embryonic stem cell cardiogenesis. Circ Res 1996; 78: 547-52.

11. Westfall MV, Samuelson LC, Metzger JM. Troponin I isoform expression is developpementally regulated in differentiating embryonic stem cell-derived cardiac myocytes. Dev Dyn 1996; 206: 24-38.

12. Ganim JR, Luo W, Ponnia S, Grupp I, Kim HW, Fergusson DG, Kadambi V, Neumann JC, Doetschman T, Kranias EG. Mouse phospholamban gene expression during development in vivo and in vitro. Circ Res 1992; 71 : 1021-30.
13. Muthuchamy M, Pajak L, Howles P, Doetschman T, Wieczorek DF. Developmental analysis of tropomyosin gene expression in embryonic stem cells and mouse embryos. Mol Cell Biol 1993; 13 : 3311-23.

14. Robbins J, Gulick J, Sanchez A, Howles $\mathrm{P}$, Doetschman T. Mouse embryonic stem cells express the cardiac myosin heavy chain genes during development in vitro. J Biol Chem 1990; 265 : 11905-9.

15. Westfall MV, Pasyk KA, Yule DI, Samuelson LC, Metzger JM. Ultrastructure and cell-cell coupling of cardiac myocytes differentiating in embryonic stem cell cultures. Cell Motil Cytoskel 1997; 36: 43-54.

16. Maltsev VA, Wobus AM, Rohwedel J, Bader M, Hescheter J. Cardiomyocytes differentiated in vitro from embryonic stem cells developmentally express cardiac-specific genes and ionic currents. Circ Res 1994; 75 : 233-44.

17. Klug MG, Soonpaa MH, Koh GY, Field LJ. Genetically selected cardiomyocytes from differentiating embryonic stem cells form stable intracardiac grafts. I Clin Invest 1996; 98: 216-24.

18. Weitzer G, Milneer DJ, Kim JU, Bradley A, Capetanaki Y. Cytoskeletal control of myogenesis: a desmin null mutation blocks the myogenic pathway during embryonic stem cell differentiation. Dev Biol 1995; 172: 422-39.

19. Li ZL, Colucci-Guyon E, Pincon-Raymond M, Mericskay M, Pournin S, Paulin D, Babinet C. Cardiovascular lesions and skeletal myopathy in mice lacking desmin. Dev Biol 1996 ; 175 : 362-6.

20. Milner DJ, Weitzer G, Tran D, Bradley A, Capetanaki Y. Disruption of muscle architecture and myocardial degeneration in mice lacking desmin. J Cell Biol 1996; 134: 1255-70.

21. Grépin C, Robitaille L, Antakly T, Nemer M. Inhibition of transcription factor GATA-4 expression blocks in vitro cardiac muscle differentiation. Mol Cell Biol 1995; 15: 4095-02.

22. Kuo CT, Morrisey EE, Anandappa R, Sigrist K, Lu MM, Parmacek MS, Soudais C, Leiden JM. GATA4 transcription factor is required for ventral morphogenesis and heart tube formation. Genes Dev 1997; 11: 1048-60.

23. Molkentin JD, Lin Q, Duncan SA, Olson EN. Requirement of the transcription factor GATA4 for heart tube formation and ventral morphogenesis. Genes Dev 1997; 11 : 1061-72.

24. Wobus AM, Guan K. Embryonic stem cell-derived cardiac differentiation: modulation of differentiation and "loss-of-function » analysis in vitro. Trends Cardiovasc Med $1998 ; 8: 64-74$.

25. Fassler R, Meyer M. Consequences of lack of $\beta_{1}$ integrin gene expression in mice. Genes Dev 1996; 9: 1896-908.

\section{TIRÉS À PART}

M. Fiszman. 